\title{
Measurement of $\phi_{s}, \Delta \Gamma_{s}$ and Lifetime in $B_{s} \rightarrow J / \psi \phi$ at ATLAS and CMS
}

\author{
Claudio Heller* \\ Excellence Cluster Universe LMU München \\ E-mail: Claudio.hellerdphysik.uni-muenchen.de \\ On behalf of the ATLAS and CMS Collaborations
}

The $B_{s} \rightarrow J / \psi \phi$ decay is characterized by the average decay width $\Gamma_{s}$ of the heavy and the light mass eigenstates, the decay width difference $\Delta \Gamma_{s}$ and by the CP violating phase $\phi_{s}$. The final state is a mixture of $\mathrm{CP}$ even and $\mathrm{CP}$ odd components that are disentangled via an angular analysis. The measurement of the decay channel provides the possibility to test the predictions of the Standard Model. In particular the measurement of the $\mathrm{CP}$ violating phase $\phi_{s}$ has the capability to unveil New Physics induced enhancement of the small Standard Model value. In this document the analyses of the $B_{s} \rightarrow J / \psi \phi$ decay with the ATLAS and CMS detectors at the LHC are presented.

14th International Conference on B-Physics at Hadron Machines April 8-12, 2013

Bologna, Italy

\footnotetext{
* Speaker.
} 


\section{Introduction}

In the decay $B_{s} \rightarrow J / \psi \phi$ channel $C P$ violation occurs via the interference of mixing and decay. This means that the $J / \psi \phi$ final state can either be reached directly in the decay of a $B_{s}^{0}$ meson or the $B_{s}^{0}$ first oscillates into a $\bar{B}_{s}^{0}$ which then decays into $J / \psi \phi$. The oscillation between the heavy $\left(B_{H}\right)$ and the light $\left(B_{L}\right)$ mass eigenstates is characterized by the mass difference $\Delta m_{s}$. The light state is expected to have a shorter lifetime than the heavy state and the Standard Model prediction for the decay width difference is $0.087 \pm 0.021 \mathrm{ps}^{-1}$ [四]. The CP violating phase $\phi_{s}$ is defined as the weak phase difference between the $B_{s}-\bar{B}_{s}$ mixing amplitude and the decay amplitude of the $b \rightarrow c \bar{c} s$ transition. The Standard Model predicts a very small value of $\phi_{s}=-0.0368 \pm 0.0018 \mathrm{rad}$ [[]]. In case of no $\mathrm{CP}$ violation, the $B_{H}$ would correspond to a $\mathrm{CP}$ odd state and $B_{L}$ to a $\mathrm{CP}$ even state.

In the $B_{s} \rightarrow J / \psi \phi$ decay the pseudoscalar $B_{s}^{0}$ decays to the two vector mesons $J / \psi$ and $\phi$. Due to conservation of total angular momentum, the relative orbital angular momentum between the $J / \psi$ and the $\phi$ can have the values $L=0,1,2$. Therefore the final state is an admixture of CP even $(L=0,2)$ and CP odd $(L=1)$ states, which are disentangled via an angular analysis. The three transversity angles $\left(\theta_{T}, \psi_{T}, \phi_{T}\right)$ [3] are defined to describe the decay topology of the final state particles $J / \psi \rightarrow \mu^{+} \mu^{-}$and $\phi \rightarrow K^{+} K^{-}$.

ATLAS and CMS both use an unbinned maximum likelihood fit to extract the physics parameters describing the decay. The differential decay rate of $B_{s} \rightarrow J / \psi \phi$ constitutes the signal part of the likelihood function which is parameterized by the mean decay width $\Gamma_{s}$, the decay width difference $\Delta \Gamma_{s}$, the mass difference $\Delta m_{s}$ and the CP violating phase $\phi_{s}$. It is expressed by the time evolution of three amplitudes and their angular dependencies. $A_{0}(t)$ and $A_{\|}(t)$ describe the $\mathrm{CP}$ even final states with $L=0,2$ and $A_{\perp}(t)$ describes the $\mathrm{CP}$ odd final state $L=1$. The decay rate also depends on the differences between the strong phases of the amplitudes $\delta_{\|}, \delta_{\perp}$ and $\delta_{0}$, where the latter is chosen to be zero in the analyses presented here.

\section{Event Reconstruction}

The reconstruction of the $B_{s}^{0}$ candidates follows a similar procedure in ATLAS and CMS. The detailed selections and cuts can be found in [ [ 4 ] for the CMS analysis and in [可] for the ATLAS measurement. In both cases the $B_{s} \rightarrow J / \psi \phi$ analysis uses measurements provided by the inner tracking detectors and the muon spectrometers. The data is selected by triggers searching for possible $J / \psi$ candidates in the event. The $J / \psi$ signature contains two oppositely charged muons with a common decay vertex and an invariant mass in a region around the world average $J / \psi$ mass. In order to reconstruct $J / \psi$ candidates all possible combinations of $\mu^{+} \mu^{-}$in the events are considered refining the invariant di-muon mass criteria. The $\phi$ candidates are formed from two additional charged tracks in the events with an invariant mass lying in a small window of $10 \mathrm{MeV}$ around the world average $\phi$ mass. The $B_{s}$ candidates are constructed from all combinations of $J / \psi$ and $\phi$ candidates in each event. Constraints on the four track common vertex fit and the $J / \psi$ invariant mass of the $B_{s}$ candidates are applied. While CMS requires the decay length of the $B_{s}$ candidates to lie between $0.02 \mathrm{~cm}$ and $0.3 \mathrm{~cm}$, ATLAS does not apply a cut on the proper 
decay time. Each $B_{s}$ candidate enters the fit with measurements of mass and decay time including corresponding uncertainties and three transversity angles.

\section{Lifetime Measurement with the CMS Detector}

The untagged CMS analysis [四] makes use of $5.0 \pm 0.1 \mathrm{fb}^{-1}$ collected in 2011 with the CMS detector in $p-p$ collisions at $\sqrt{s}=7 \mathrm{TeV}$. The data sample contains $14456 B_{s}$ candidates in the mass range $(5.24-5.49 \mathrm{GeV}$. An unbinned maximum likelihood fit is performed extracting the physics parameters $\Delta \Gamma_{s}, \Gamma_{s},\left|A_{\perp}(0)\right|^{2},\left|A_{0}(0)\right|^{2}$ and $\delta_{\|}$. The third amplitude $\left|A_{\|}(0)\right|^{2}$ is constrained by the normalization $\left|A_{0}(0)\right|^{2}+\left|A_{\perp}(0)\right|^{2}+\left|A_{\|}(0)\right|^{2}=1$. No CP violation in the neutral $B_{s}$ system is assumed in the fit which translates to set $\phi_{s}$ to zero.

The signal component of the likelihood function consists of the normalized untagged decay rate [四], describing the decay time and angular dependence, and the mass is parameterized by the sum of two Gaussians. To take the limited detector resolution into account, the decay rate is smeared with the per-event decay time uncertainty. Detector effects on the angular distributions and decay time efficiency are determined from MC simulation and included in the signal description. The background is modeled by an exponential function for the mass, and two Gaussians and two exponential functions for the proper decay time. Two angles $\left(\cos \theta_{T}\right.$ and $\left.\cos \psi_{T}\right)$ of the background component are described by a series of Legendre polynomials and $\phi_{T}$ is modeled using a sinusoidal distribution.

Several parameters of the fit model are determined in advance and kept fix in the maximum likelihood fit with the complete likelihood function. In a first step the mean and the narrower width of the two Gaussians are determined in a mass only fit. The parameters describing the background angles are measured in a fit to data in the $B_{s}$ mass-sideband distributions. A calibration scale factor of the proper decay time uncertainty is determined in a mass lifetime fit on data without the selection cut on the decay length. In the last step the full maximum likelihood fit is performed taking into account the signal and background models for mass, time and angular distributions.

Mass and proper decay time projections of the fit are shown in figure $\mathbb{~} \mathbf{l}$. The following values for the physics parameters of interest are extracted [四]:

$$
\begin{aligned}
\Delta \Gamma_{s} & =0.048 \pm 0.024 \text { (stat.) } \pm 0.003 \text { (syst.) } \mathrm{ps}^{-1} \\
\tau_{B_{s}} & =0.04580 \pm 0.00059 \text { (stat.) } \pm 0.00022 \text { (syst.) } \mathrm{cm} \\
\left|A_{0}(0)\right|^{2} & =0.528 \pm 0.010 \text { (stat.) } \pm 0.015 \text { (syst.) } \\
\left|A_{\perp}(0)\right|^{2} & =0.251 \pm 0.013 \text { (stat.) } \pm 0.014 \text { (syst.) } \\
\delta_{\|} & =2.79 \pm 0.14 \text { (stat.) } \pm 0.19 \text { (syst.) rad }
\end{aligned}
$$

The dominant systematic uncertainties for the decay width difference $\Delta \Gamma_{s}$ are the modeling of the signal time resolution, the background angels and the parameterization of the proper decay time efficiency. The decay time efficiency is also, together with the modeling of the signal mass, the largest systematic of the lifetime $\tau_{B_{s}}$. For the amplitudes the dominant systematics are the assumption that there is no $\mathrm{S}$-wave component in the data sample and the statistics and parameterization of the angular efficiency. A detailed break down of the systematic uncertainties can be found in [四]. 

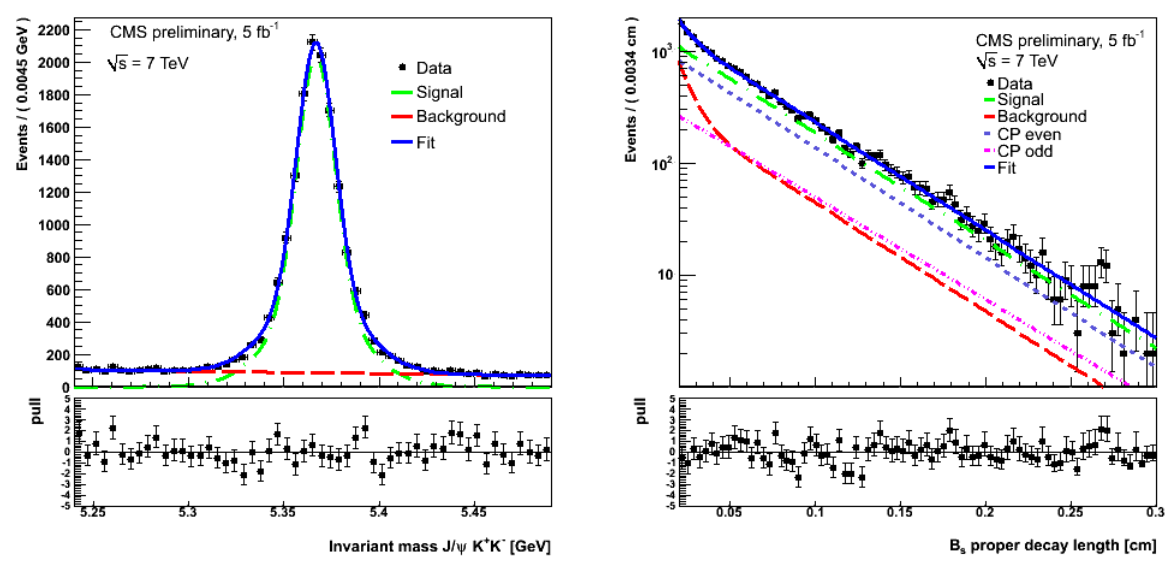

Figure 1: Mass (left) and proper decay time (right) projections of the maximum likelihood fit to the CMS data [䧃]. Black points represent the data, the solid blue line shows the full fit, the dashed green line is the signal component and the dashed red line is background. CP even and odd component of the signal decay time are shown as blue short dashed and magenta dash-dot-dotted lines. The histogram below shows the corresponding pull distribution between the data and the fit.

\section{Tagged $B_{S} \rightarrow J / \psi \phi$ Analysis with the ATLAS Detector}

ATLAS has updated its untagged analysis of the $B_{s} \rightarrow J / \psi \phi$ decay published in 2012 [ 6 ] with the application of initial state flavor tagging [5]. The measurement is performed using $4.9 \mathrm{fb}^{-1}$ of data collected in 2011 with the ATLAS detector in $p-p$ collisions with $\sqrt{s}=7 \mathrm{TeV}$. No cut on the proper $B_{s}$ decay time is applied resulting in about $130000 B_{s}$ candidates in the mass range of $5.15 \mathrm{GeV}<m\left(B_{s}^{0}\right)<5.65 \mathrm{GeV}$.

The differential decay rate describing the signal decay includes terms for the S-wave decays $B_{S} \rightarrow$ $J / \psi f_{0}$ and non-resonant $B_{s} \rightarrow J / \psi K^{+} K^{-}$. The S-wave component is a pure CP odd state parameterized by an additional amplitude $\left|A_{s}(0)\right|$ and a related strong phase $\delta_{s}$. The four amplitudes are normalized to one: $\left|A_{0}(0)\right|^{2}+\left|A_{\perp}(0)\right|^{2}+\left|A_{\|}(0)\right|^{2}+\left|A_{s}(0)\right|^{2}=1$. The normalized tagged differential decay rate [G] constitutes the time and angular dependent part of the signal component of the likelihood function. To take the detector resolution into account it is convoluted with a Gaussian resolution function making use of the event-by-event decay time uncertainty. The sculpting of the angular distributions by the detector is accounted for applying a four-dimensional binned acceptance method that provides an efficiency depending on the transversity angles and the $p_{T}$ of the $B_{s}$ candidates. A single Gaussian function smeared with an event-by-event mass resolution models the signal mass. The background mass is modeled by a linear function and the proper decay time of the background is described by the sum of a Gaussian, two positive exponentials and a negative exponential for events with poor decay time resolution. The background angular model is described by functions that were empirically determined from sideband data. The likelihood function includes additional terms for the contamination of the data sample with peaking background from $B_{d}$ reflections. The decays $B_{d} \rightarrow J / \psi K^{*}$ and non-resonant $B_{s} \rightarrow J / \psi K \pi$ can be identified as $B_{s}$ candidates if the final state pion is mis-reconstructed as a kaon. Their contribution to the data sample and their mass, decay time and angular shapes are determined using MC simulation and the corresponding parameters are kept fix in the fit. In total the fit features 25 free parameters including the physics 
parameters of interest $\phi_{s}, \Delta \Gamma_{s}$ and $\Gamma_{s}$.

Flavor tagging enters the fit in form of a probability that the $B_{s}$ candidate is a particle or antiparticle. ATLAS uses two methods for opposite side flavor tagging which are both studied and calibrated using the $B^{ \pm} \rightarrow J / \psi K^{ \pm}$decay in data.

The first flavor tagging method is the muon cone charge tagger using muons from semi-leptonic

\begin{tabular}{c|c|c|c} 
Tagger & Efficiency [\%] & Dilution [\%] & Tagging Power [\%] \\
\hline Segment Tagged muon & $1.08 \pm 0.02$ & $36.7 \pm 0.7$ & $0.15 \pm 0.02$ \\
Combined muon & $3.37 \pm 0.04$ & $50.6 \pm 0.5$ & $0.86 \pm 0.04$ \\
Jet charge & $27.7 \pm 0.1$ & $12.68 \pm 0.06$ & $0.45 \pm 0.05$ \\
\hline Total & $32.1 \pm 0.1$ & $21.3 \pm 0.08$ & $1.45 \pm 0.05$
\end{tabular}

Table 1: Table showing the performance of the flavor tagging algorithms [[]]. While segment tagged muons consist of a full inner detector track that is matched to track segments in the muon spectrometer, Combined muons have a full track in the inner detector and in the muon spectrometer.

$B$ decay on the opposite side. The events are searched for an additional muon originating near the primary interaction. If there is more than one additional muon, the one with the highest $p_{T}$ is chosen. A muon cone charge variable is calculated from tracks found in the inner tracking detectors that are in a cone of $\Delta R<0.5$ around the momentum axis of the muon. The muon cone charge is constructed as sum of the charge of the tracks weighted with their transverse momentum and a tag probability is derived from that.

The second tagging method is the jet charge tagger. In the absence of a muon, a b-tagged jet reconstructed using the "anti- $k_{T}$ " algorithm is required in the event. Jets and tracks which are close to the signal momentum axis are not taken into account. The tag probability is derived from the jet charge which is a sum of the charge of the tracks in the jet weighted with their transverse momentum. The performance of the flavor tagging algorithms is shown in table $\mathbb{W}$. The following values have been obtained for the physics parameters in the ATLAS analysis [[]]:

$$
\begin{aligned}
\phi_{s} & =0.12 \pm 0.25 \text { (stat.) } \pm 0.11 \text { (syst.) rad } \\
\Delta \Gamma_{s} & =0.058 \pm 0.021 \text { (stat.) } \pm 0.009 \text { (syst.) } \mathrm{ps}^{-1} \\
\Gamma_{s} & =0.677 \pm 0.007 \text { (stat.) } \pm 0.003 \text { (syst.) } \mathrm{ps}^{-1} \\
\left|A_{0}(0)\right|^{2} & =0.529 \pm 0.006 \text { (stat.) } \pm 0.011 \text { (syst.) } \\
\left|A_{\|}(0)\right|^{2} & =0.220 \pm 0.008 \text { (stat.) } \pm 0.009 \text { (syst.) } \\
\left|A_{S}(0)\right|^{2} & =0.024 \pm 0.014 \text { (stat.) } \pm 0.028 \text { (syst.) } \\
\delta_{\perp} & =3.89 \pm 0.46 \text { (stat.) } \pm 0.13 \text { (syst.) rad }
\end{aligned}
$$

The dominant systematic uncertainty for $\phi_{S}$ is the error of the tag probability which is caused by statistical fluctuations of the $B^{ \pm} \rightarrow J / \psi K^{ \pm}$calibration sample. For all other parameters the modeling of the background angles constitutes the largest systematic uncertainty. Compared to the untagged fit [目] the statistical uncertainty of $\phi_{s}$, which was measured with $\phi_{s}=0.21 \pm 0.41$ (stat.) \pm 0.10 (stat.) rad has decreased by $40 \%$. The obtained values and uncertainties of the parameters are 
consistent with the untagged analysis and $\phi_{s}$ and $\Delta \Gamma_{s}$ are in agreement with the Standard Model. The likelihood contour plot in the $\phi_{s}-\Delta \Gamma_{s}$ plane for the tagged analysis is shown in figure $\square$.

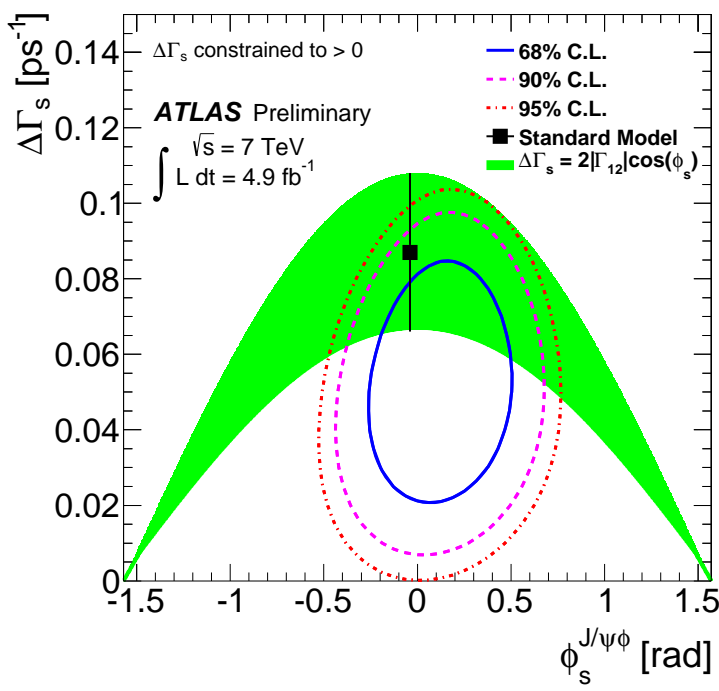

Figure 2: Two-dimensional likelihood scan in the $\phi_{s}-\Delta \Gamma_{s}$ plane [四]. Only statistical uncertainties are taken into account.

\section{Summary}

ATLAS and CMS have performed analyses of the decay $B_{s} \rightarrow J / \psi \phi$ measuring $\Gamma_{s}$ and $\Delta \Gamma_{s}$. In addition ATLAS has measured the CP violating phase $\phi_{s}$. All results are in agreement with previous measurements and with the Standard Model. Since the statistical uncertainties still dominate for the essential parameters, future updates of the analyses including the usage of data collected in 2012 and enhancements of the analysis methods, have the capability to improve the precision of the results.

\section{References}

[1] A. Lenz and U. Nierste, Numerical Updates of Lifetimes and Mixing Parameters of B Mesons, arXiv:1102.4274 [hep-ph].

[2] M. Bona et al., Constraints on new physics from the quark mixing unitarity triangle, Phys.Rev.Lett. 97 (2006) 151803 [hep-ph/0605213].

[3] A. S. Dighe, I. Dunietz and R. Fleischer, Extracting CKM phases and $B_{s}-\bar{B}_{s}$ mixing parameters from angular distributions of nonleptonic B decays, Eur.Phys.J.C 6 (1999) 647 [hep-ph/ 9804253 ].

[4] CMS Collaboration, Measurement of the Bs lifetime difference, CMS-PAS-BPH-11-006.

[5] ATLAS Collaboration, Flavour tagged time dependent angular analysis of the $B_{s} \rightarrow J / \psi \phi$ decay and extraction of $\Delta \Gamma$ and the weak phase $\phi_{s}$ in ATLAS, ATLAS-CONF-2013-039.

[6] ATLAS Collaboration, Time-dependent angular analysis of the decay $B_{S} \rightarrow J / \psi \phi$ and extraction of $\Delta \Gamma_{s}$ and the CP-violating weak phase $\phi_{s}$ by ATLAS, JHEP 1212 (2012) 072 [arXiv: 1208.0572 [hep-ex]]. 